Published in final edited form as:

Neurocase. 2010 August ; 16(4): 307-316. doi:10.1080/13554790903559671.

\title{
fMRI tracks reductions in repetitive behaviors in autism: Two case studies
}

\author{
Gabriel S. Dichter ${ }^{1,2,3,4}$, Linmarie Sikich ${ }^{1,2}$, Steve Mahorney ${ }^{4}$, Jennifer N. Felder ${ }^{2}$, Kristen \\ S. L. Lam ${ }^{2}$, Lauren Turner-Brown ${ }^{2}$, and James Bodfish ${ }^{1,2,5}$ \\ ${ }^{1}$ Department of Psychiatry, University of North Carolina at Chapel Hill School of Medicine, Chapel \\ Hill, NC, USA \\ ${ }^{2}$ Carolina Institute for Developmental Disabilities, University of North Carolina at Chapel Hill \\ School of Medicine, Chapel Hill, NC, USA \\ ${ }^{3}$ Duke-UNC Brain Imaging and Analysis Center, Duke University Medical Center, Durham, NC, \\ USA \\ ${ }^{4}$ Department of Psychiatry and Behavioral Sciences, Duke University Medical Center, Durham, \\ NC, USA \\ ${ }^{5}$ Center for Development and Learning, University of North Carolina at Chapel Hill, Chapel Hill, \\ NC, USA
}

\begin{abstract}
Autism is characterized by abnormal prefrontal brain activation during cognitive control, a potential biomarker of repetitive behaviors. In this proof-of-principle study, functional magnetic resonance imaging (fMRI) was used to examine brain activity during an oddball task in two highfunctioning males with autism before and after 12 weeks of treatment with citalopram, a selective serotonin reuptake inhibitor. One participant showed marked reductions in repetitive behaviors whereas the other showed mild worsening. Brain activation in relevant prefrontal regions increased in only the participant whose repetitive behavior symptoms improved. These findings suggest that fMRI may elucidate potential mechanisms of action of targeted autism interventions.
\end{abstract}

\section{Keywords}

Autism; Asperger's Disorder; Citalopram; Celexa; Repetitive behaviors; Target detection; Oddball; Cingulate gyrus; fMRI; Functional magnetic resonance imaging

\section{INTRODUCTION}

The goal of this report is to demonstrate proof-of-principle that investigations of neurofunctional changes in the context of treatment of repetitive behaviors (RBs) in autism are feasible and hold promise for understanding the potential mechanisms of action of autism interventions. Changes in brain activation and RBs were assessed in two individuals with Autistic Disorder before and after treatment with citalopram, a selective serotonin reuptake inhibitor, administered in a double-blind context. We focused specifically on

(C) 2010 Psychology Press, an imprint of the Taylor \& Francis Group, an Informa business

Address correspondence to Dr Gabriel S. Dichter, Department of Psychiatry, University of North Carolina School of Medicine, CB\# 3366, 101 Manning Drive, Chapel Hill, NC 27599-3366, USA. (dichter@ med.unc.edu.).

The authors have no conflicts of interest to declare. 
changes in prefrontal activation because of prior neuroimaging evidence of linkages between prefrontal functioning and RBs in autism (e.g., Shafritz, Dichter, Baranek, \& Belger, 2008) as well as other disorders characterized by RBs (Gu et al., 2008; Marsh, Zhu, Wang, Skudlarski, \& Peterson, 2007).

The premise of the present case study design is that (1) RBs in autism may be mechanistically linked to the functions of the frontal lobes (Russell, 1997), (2) frontal lobe functions may be assessed via 'executive' tasks administered in a functional magnetic resonance imaging (fMRI) context (e.g., Schmitz et al., 2006; Shafritz et al., 2008; Thakkar et al., 2008), and, in turn (3) reductions in RBs due to a psychopharmacologic intervention should be evident in fMRI outcomes that may ultimately prove to be a biomarker of RB symptom reductions in autism.

Repetitive behaviors are a core feature of autism (American Psychiatric Association, 1994) and refer to behaviors linked by repetition, rigidity, invariance, and inappropriateness (Kanner, 1943; Lewis \& Bodfish, 1998). The 'frontostriatal hypothesis' of autism implicates the basal ganglia and its afferents to the prefrontal cortex (Robbins, 1997) and is supported by both neuropsychological and animal studies (Insel, 1992; Lewis, Gluck, Beauchamp, Keresztury, \& Mailman, 1990; Masterman \& Cummings, 1997; Robbins, Mittleman, O'Brien, \& Winn, 1990; Visser, Bar, \& Jinnah, 2000). Though functional pathology of the basal ganglia has not been directly observed in autism, recent structural imaging data suggest differential developmental trajectories of the caudate nucleus and putamen in autism (Langen et al., 2009), regions that both have extensive connections to prefrontal brain structures (Cummings, 1993).

There is a host of neuroimaging evidence indicating anomalous activation of prefrontal brain regions in individuals with autism during 'cognitive control' or 'executive' tasks (see Dichter, Felder, \& Bodfish, 2009, for a review). The specific prefrontal regions affected and the direction of changes are heterogeneous across studies and related to the specific cognitive tasks utilized, supporting the widely held view that executive functions include a number of distinct cognitive processes. Hyperactivation in the left middle and inferior frontal gyri and orbitofrontal cortex has been reported during a go/no-go (i.e., motor inhibition) task, whereas hyperactivation of the left insula was observed in the same participants during a spatial Stroop (i.e., interference inhibition) task (Schmitz et al., 2006). Hyperactivation in the right superior, middle and inferior prefrontal cortex and the left inferior parietal cortex has been reported during an auditory novelty detection task (Gomot, Belmonte, Bullmore, Bernard, \& Baron-Cohen, 2008). Rostral anterior cingulate cortex hyperactivation during an antisaccade task that predicted the magnitude of repetitive behaviors was reported by Thakkar et al. (2008). Finally, hypoactivation bilaterally in middle frontal, right superior frontal and left inferior frontal regions has been reported during a Tower of London (i.e., problem solving) task (Just, Cherkassky, Keller, Kana, \& Minshew, 2007).

Although there is no direct evidence linking striatal dysfunction to RBs in autism, our research group reported hypoactivation in the striatum, as well as in the middle and inferior frontal gyri and anterior cingulate cortex (ACC) in autism during a standard visual target detection (i.e., oddball) task (Shafrtiz et al, 2008). Furthermore, the magnitude of ACC activation inversely correlated with RBs in the autism sample. The ACC circuit is one of five parallel circuits that link the frontal lobes with subcortical structures. The ACC circuit projects to the ventral striatum, then to the ventral and rostrolateral globus pallidus and rostrodorsal substantia nigra, that, in turn, project to the medial dorsal nucleus of the thalamus, and then the circuit is completed via projections to the ACC (for a review, see Cummings, 1993). Injury to this circuit produces deficits on response inhibition tasks 
(Drewe, 1975), and other repetitive behavior disorders, such as Tourette's Syndrome and obsessive compulsive disorder, are characterized by dysfunction within this circuit (Baxter et al., 1987; Singer, Hahn, \& Moran, 1991).

The purpose of the present study was to demonstrate proof-of-principle that changes in prefrontal activation in individuals with autism may be a sensitive biomarker of reductions in RB symptoms due to psychopharmacologic intervention. Citalopram, a selective serotonin reuptake inhibitor (SRI), was chosen because SRIs are widely used for the treatment of RBs in autism and positive results of SRIs have been demonstrated in controlled pilot studies (Buchsbaum et al., 2001; Hollander et al., 2005; McDougle et al., 1996). Although a recent large-scale trial did not find differences between citalopram and placebo in effects on RBs in autism spectrum disorders (King et al., 2009), it is clear that there is wide heterogeneity in response to autism interventions, and fMRI may elucidate potential sources of such variability. We utilized a visual oddball target-detection task previously demonstrated to be sensitive to prefrontal dysfunction in individuals with autism (Shafritz et al., 2008) to investigate whether changes in prefrontal activation parallel changes in RB symptoms in response to treatment. Our primary hypothesis was that successful treatment of RBs would be associated with increased activity in the prefrontal cortex, specifically the middle and inferior frontal gyri and in the ACC.

\section{METHOD}

\section{Participants}

The two participants in the present study were the first two entrants into a larger, ongoing, double-blind randomized controlled trial comparing citalopram to placebo in adults with diagnoses of Autistic Disorder or Asperger's Disorder and moderate-to-high levels of RBs as defined by baseline Yale-Brown Obsessive Compulsive Scale - Pervasive Developmental Disorder Version (CYBOCS-PDD, Scahill et al., 2006) scores of eight or higher. Both participants were randomized to the active study arm, were right-handed, male, had normal vision, a DSM-IV (American Psychiatric Association, 1994) clinical diagnosis of Autistic Disorder informed by spectrum scores on the Autism Diagnostic Observation ScheduleGeneric (Lord et al., 2000), were high-functioning, had no history of psychiatric illness other than autism, and consented to a protocol approved by the local Human Investigations Committees at both UNC-Chapel Hill and Duke University Medical Centers. Blinds were not broken to study participants until completion of both imaging sessions and all studyrelated assessments. Image analysts were blind to group membership (i.e., citalopram or placebo) as well. Participants were paid $\$ 50$ for completing the imaging portion of the study.

Exclusion criteria included: (1) history of intolerable adverse effects with two or more SSRIs that are not attributable to inappropriate dosing, dose escalation, concomitant treatments or developmental stage; (2) history of an exposure to citalopram/escitalopram of sufficient dose or duration to determine response status; (3) history of failed adequate clinical trials of two SSRIs; (4) anticonvulsant medication use; (5) history of gestational age $<34$ weeks, birth weight $<2000 \mathrm{~g}$, or intraventricular hemorrhage; (6) history of known medical condition associated with autism including Fragile $\mathrm{X}$ syndrome, tuberous sclerosis, neurofibromatosis, phenylketonuria, epilepsy and gross brain injury; (7) MRI contraindication; (8) concomitant psychotropic use; and (9) intelligence scores $<70$ as assessed by the Weschler Abbreviated Scale of Intelligence (WASI) (Weschler, 1999).

Although both participants demonstrated criterion levels of RBs and had functional impairments related to their RBs, neither experienced subjective distress as a result of these RBs. The first study entrant, 'Case 1', was a 22-year-old college student who lived independently. His most debilitating RB was excessive editing and referencing of class 
assignments that led to a host of functional impairments at school. The second study entrant, 'Case 2', was a 19-year-old high-school student who lived with his parents. His most debilitating RBs involved excessive daily routines and touching rituals.

\section{Treatment}

Participants were treated by psychiatrists who specialize in medication management of individuals with autism and other neurodevelopmental disorders. Citalopram doses were initiated at $5 \mathrm{mg} /$ day and were flexibly adjusted after considering whether the clinical response was sufficient as defined by a Clinical Global Impressions - Improvement Score (CGI-I, Guy, 1976) of ' 1 - very much improved' or ' 2 - much improved' and whether there were significant adverse effects.

Case 1 attained a dosage of $40 \mathrm{mg} /$ day by week 4 , and maintained that dosage (i.e., steady dosage duration $=8$ weeks) until follow-up fMRI scan 98 days after his pre-treatment fMRI scan. The second study entrant, Case 2, attained a dosage of $10 \mathrm{mg} /$ day during the second week and remained at that dosage for 4 weeks, then increased to $20 \mathrm{mg} /$ day for 4 weeks, and then increased to $30 \mathrm{mg} /$ day for the remaining 4 weeks (i.e., steady dosage duration $=4$ weeks) until follow-up fMRI scan 91 days after his pre-treatment fMRI scan.

Case 1 reported mild difficulties with concentration and dizziness during his first day of treatment that resolved quickly after taking his pills in the afternoon rather than the morning. $\mathrm{He}$ also reported moderate amotivation starting between weeks 4 and 8 that lasted until the end of his participation, as well as mild fatigue, beginning at week 8, that lasted until the end of his participation. Case 2 reported no side effects.

\section{fMRI task}

Each fMRI session consisted of a visual target detection task that included six task runs, each containing 160 stimuli presented centrally for $500 \mathrm{~ms}$ with an interstimulus interval jittered between 1000 and $2500 \mathrm{~ms}$. There were three stimulus categories: squares, circles, and triangles of various colors and sizes. At the start of each run, participants were instructed both verbally and via an instruction screen (e.g., 'Target $=\mathbf{0}$ ') which stimulus category would be the 'target' on that run. Each run included three types of stimuli: (1) Frequently occurring 'Standard' stimuli that occurred on $87.5 \%$ of trials; (2) Infrequently occurring 'Novel' stimuli that occurred on $6.25 \%$ of trials; and (3) Infrequently occurring oddball 'Target' stimuli that occurred on $6.25 \%$ of trials. The stimuli designated as target, novel, and standard changed every run. Each participant viewed 10 target events per run, resulting in 60 target stimuli in the six runs analyzed. Participants were instructed to respond as quickly and accurately as possible, and to press one button, with their right hand, for all non-target stimuli and an alternate button for all target stimuli.

\section{Imaging methods}

Scanning was performed on a General Electric Health Technologies, 3 Tesla Signa Excite HD scanner system with 50-mT/m gradients (General Electric, Waukesha, WI, USA). Head movement was restricted using foam cushions and Velcro straps. An eight-channel head coil was used for parallel imaging. Sixty-eight high resolution images were acquired using a 3D fast SPGR pulse sequence $\left(\mathrm{TR}=500 \mathrm{~ms} ; \mathrm{TE}=20 \mathrm{~ms} ; \mathrm{FOV}=24 \mathrm{~cm}\right.$; image matrix $=256^{2}$; voxel size $=0.9375 \times 0.9375 \times 1.9 \mathrm{~mm}$ ) and used for coregistration with the functional data. These structural images were aligned in the near axial plane defined by the anterior and posterior commissures. Whole brain functional images consisted of 34 slices parallel to the AC-PC plane using a BOLD-sensitive gradient-echo sequence with spiral-in k-space sampling and SENSE encoding to take advantage of the eight-channel coil, at TR of 1500 
ms (TE: $27 \mathrm{~ms}$; FOV: $25.6 \mathrm{~cm}$; isotropic voxel size: $4 \mathrm{~mm}^{3}$; SENSE factor = 2). Runs began with 4 discarded RF excitations.

\section{Imaging data analysis}

Functional data were preprocessed using FSL version 4.0.2 (Oxford Centre for Functional Magnetic Resonance Imaging of the Brain (FMRIB), Oxford University, UK].

Preprocessing was applied in the following steps: (i) brain extraction (Smith et al., 2004), (ii) motion correction using MC FLIRT (Smith, 2002), (iii) spatial smoothing using a Gaussian kernel of FWHM $5 \mathrm{~mm}$, (iv) mean-based intensity normalization of all volumes by the same factor, and (v) high-pass filtering (Jenkinson, Bannister, Brady, \& Smith, 2002). All registrations were carried out using an intermodal registration tool (Jenkinson et al., 2002; Smith et al., 2004). Voxel-wise temporal autocorrelation was estimated and corrected using FMRIB's Improved Linear Model (Jenkinson \& Smith, 2001). Onset times of events were used to model signal responses containing regressors for all event types which were convolved with a double- $\gamma$ function to model the hemodynamic response. Activation images were calculated by a mixed effects higher-level analysis using Bayesian estimation techniques, FMRIB Local Analysis of Mixed Effects (FILM, Woolrich, Ripley, Brady, \& Smith, 2001) with cluster mean threshold of $Z>1.5$ and a cluster-corrected significance threshold of $p<.05$ (FLAME 1+2, Beckmann, Jenkinson, \& Smith, 2003). Comparisons of Time 1 and Time 2 scans were conducted by a voxelwise mixed-effects analysis using FLAME $1+2$. We thresholded $z$-score images using clusters determined by $z>1.7$ and a (corrected) cluster significance threshold of $p=.05$. A two-sample paired $t$-test identified group mean differences.

\section{RESULTS}

\section{Treatment outcomes}

Table 1 illustrates intelligence and treatment symptoms scores for both participants. On the CYBOCS-PDD, the primary outcome measure, Case 1 demonstrated a $27 \%$ increase relative to baseline in severity of RB symptoms, whereas Case 2 showed a $38 \%$ decrease in severity of RB symptoms. Case 1 had 'minimally worse' functioning, whereas Case 2 had 'minimally improved' functioning as assessed by their CGI-I scores. Clinically, Case 1 reported that treatment somewhat reduced social anxiety but did not reduce his compulsive behaviors with class assignments. Moreover he reported that treatment reduced his motivation, made him 'apathetic', reduced his focus and attention to detail, and caused his academic performance to decline. Case 2 reported that treatment reduced his need to complete interfering daily routines and that transitions became generally easier after treatment.

\section{fMRI behavioral performance}

Figure 1 indicates accuracy and reaction times (RT) for both participants at both timepoints for each stimulus category. The effect sizes for changes in RTs and accuracy to target stimuli for both cases ranged from .07 to .22 , representing small effect sizes.

\section{Imaging data}

Analyses of functional imaging data included only epochs corresponding to correct responses to ensure that brain activation values did not reflect differential task performance. Figure 2 illustrates activation changes to target stimuli, and Table 2 indicates MNI coordinates and intensities of activations. These results illustrate that Case 1 did not show evidence of increased activation in relevant prefrontal regions; although he demonstrated increased supplementary motor cortex activation after treatment, this region is not part of the 
frontal-subcortical circuitry previously implicated as abnormal in autism. However, Case 2 showed significantly greater activation in a number of relevant prefrontal regions posttreatment, most notably in the ACC (Brodmann's Area 32).

\section{DISCUSSION}

To our knowledge, there are no published accounts of the neurofunctional effects of autism interventions. Our goal in this proof-of-principle case study was to demonstrate the feasibility and potential promise of investigations of neurofunctional alterations in the context of specific behavioral changes in response to particular treatments in autism. We employed a visual target-detection task that has been shown previously to elicit abnormal prefrontal activation in individuals with autism (Shafritz et al., 2008).

Case 2 demonstrated marked improvements in RBs as assessed by the primary outcome measure, the CYBOCS-PDD, whereas Case 1 did not show CYBOCS-PDD improvement. Specifically, CYBOCS-PDD scores of Case 2 decreased from 16 to 10, a clinically significant decrease. In a sample of 172 medication-free children with pervasive developmental disorder, CYBOCS-PDD score averages were 14.4, with a standard deviation of 3.86 (Scahill et al., 2006). Thus, CYBOCS-PDD scores of Case 2 dropped approximately 1.5 standard deviations, and changed from approximately one-half standard deviation above the mean to approximately one standard deviation below the mean. Of note, CYBOCS-PDD change in Case 2 did not parallel his CGI-I scores, suggesting that the effects of citalopram in Case 2 may have been constrained to changes in RBs, but not of sufficient global impact on overall functioning to reflect a large change in his CGI-I score.

Functional brain imaging data indicated that improvements in RB symptoms in Case 2 were accompanied by increased activation in a number of prefrontal regions, with greatest increased activation in the ACC (Broadmann's Area 32). In contrast, there were no changes in the activity of the ACC or other relevant prefrontal regions in Case 1 who showed no reduction in RBs. We interpret increased activation of the ACC and other prefrontal regions in Case 2 potentially to reflect compensatory mechanisms that were engaged during performance of the target detection task after effective treatment. We interpret the lack of increased activation in these regions in Case 1 to reflect ineffective treatment. The increase in supplementary motor cortex activation in Case 1 may reflect the observed increase in RBs after citalopram treatment in this individual.

The direction of fMRI effects in the present context (i.e., greater prefrontal activation that corresponded with RB reductions in Case 2) warrants replication, particularly in light of a wealth of data demonstrating that autism is associated with relatively increased prefrontal activation during cognitive control (see Dichter et al., 2009 for a review). In particular, Thakkar et al. (2008) reported a significant correlation between increased ACC activation during an antisaccade task and increased RBs. As indicated above, in the present context, prefrontal activation increases may be conceptualized to reflect compensatory mechanisms that emerge soon after initiation of effective treatment that are not necessarily inconsistent with a pattern of general prefrontal hyperactivation during executive tasks in autism. Manoach (2003), in a review of an analogous issue in the schizophrenia literature, has suggested that variability in behavior and brain activation may best be regarded as intrinsic to heterogeneous disorders. Thus, we interpret the present findings within the framework of dysregulated and inefficient frontostriatal recruitment in autism during cognitive control tasks that is altered with citalopram-induced reductions in RB symptoms.

The ACC has a number of functions, but most specifically mediates overcoming prepotent responses (e.g., Devinsky, Morrell, \& Vogt, 1995) and online monitoring of errors in the 
presence of response conflict (MacDonald, Cohen, Stenger, \& Carter, 2000). In the present context, reductions in RBs, a real-world form of overcoming prepotent behavioral tendencies, may coincide with increased ACC activity while overcoming prepotent responses in the context of a target detection task.

More broadly, these neuroimaging findings offer unique information about the potential mechanisms of action of citalopram-induced RB symptom reductions. Changes in questionnaire-based measures of RBs in the context of a clinical trial may be due to what Braunholtz, Edwards, and Lilford (2001) have summarized as 'trial effects', that is, the effects of participating in a clinical trial rather than the direct effects of a treatment. Such effects may include treatment effects, placebo effects, Hawthorne effects, and observer effects. Although such effects cannot definitely be ruled out in the present context, neuroimaging data may indicate that the benefits of a treatment are due to changes in functioning of relevant brain regions rather than such other factors.

We note a number of methodological features which warrant interpretive caution. The final citalopram dosage differed between participants. Case 1 experienced adverse effects (i.e., amotivation and mild fatigue) that may have compromised his performance and patterns of brain activation during the post-treatment oddball task, whereas Case 2 did not report adverse effects. Thus, it is possible that the differences in brain activation may reflect changes in RBs as well as differences in motivation. Differences in reaction times between pre-and post-treatment scans (i.e., Case 1, but not Case 2, showed increased reaction times) is a potential confound as well that may be addressed in future trials with adequate sample sizes that allow for including reaction time as a covariate in imaging analyses. The difference in RB type may have moderated treatment response as well, a possibility that will be evaluated in future, larger-scale studies. Additionally, the relatively long intervening time period between scans suggests that fMRI changes may not have been due solely to citalopram.

Regarding the analysis of fMRI data, we used a relatively liberal statistical threshold for change in these case reports, and future studies with larger samples will use more conservative approaches. Moreover, analyses in case studies are descriptive and qualitative. Firm inferences about linkages between changes in prefrontal activation and changes in RBs in response to treatment will require an examination of group-level data from the larger trial. We also note that the case study approach does not suggest conclusively a linkage between changes in RBs and changes in fMRI activation, and we interpret the present findings as supporting but not sufficient evidence of a causal link between changes in fMRI activation and changes in RB symptom severity.

Additionally, analyses focused on fMRI response to target events without controlling for responses to novel events, an approach that has been employed in group-level fMRI studies of set shifting in autism (i.e., Gomot et al., 2008, Shafritz et al., 2008). In this regard, it cannot be firmly concluded that neural responses were target-specific. This approach was used because the case study design did not yield sufficient statistical power to detect significant interaction effects (i.e., Time $\mathrm{X}$ [target vs. novel] effects), even at a liberal statistical threshold. Thus, responses to target events only were examined. However, we note that contrasting Time 2 and Time 1 scans controlled for each participant's pre-treatment responses to target events, though this across-scan correction is clearly less optimal than a within-scan correction.

Finally, the poor accuracy of both patients restricted the number of trials analyzed. We note that Shafritz et al. (2008) reported results from a highly similar visual oddball task where participants with autism spectrum disorders viewed 55 target events and achieved only $42 \%$ 
accuracy (i.e., an average of 23 correct responses). These rates are roughly comparable to the present context, where Case 2 achieved accuracies of 38\% and 52\% at timepoints 1 and 2 (i.e., 23 and 31 correct responses to target events, respectively) and Case 1 achieved accuracies of $35 \%$ and $21 \%$ at timepoints 1 and 2 (i.e., 21 and 13 correct responses to target events, respectively). Additionally, we note that event-related fMRI psychiatry studies have utilized far lower numbers of events than analyzed in the present study (e.g., Forbes et al., 2009).

These two cases clearly demonstrate that fMRI studies are feasible and can be utilized longitudinally in individuals with autism who are treated with medication. These cases also demonstrate the importance of distinguishing between treatment responders and nonresponders when assessing potential mechanisms of treatment action. These results also raise the intriguing possibility that increases in prefrontal brain activation during a visual oddball task may be a sensitive biomarker of reductions in RBs in autism. Though the utility of brain imaging is clearly limited in clinical settings, these data suggest that brain imaging is an invaluable research tool to elucidate neurobiological mediators of autism interventions. In summary, although by no means a definitive assessment of potential treatment mechanisms, the present case study results are consistent with the hypothesis that reductions in RBs in autism in response to treatment may be related to compensatory increases in activation of the ACC and other pre-frontal cortical brain regions, a pattern of findings that holds promise as a potential biomarker of treatment response in autism.

\section{Acknowledgments}

The authors would like to thank Josh Bizzell, Chris Petty, Todd Harshbarger, and Syam Gadde for assistance with image analysis, MRI technologists Susan Music and Natalie Goutkin for assistance with data acquisition, and Jamie Clary and Vicki Harper for assistance with clinical data collection. This research was supported by the Dana Foundation (Dichter), a career development award from UNC-Chapel Hill, NIH/NCRR K12 RR023248 (Orringer), and NIMH K23 MH081285 (Dichter). Assistance for this study was provided by the Neuroimaging Core of the UNC Neurodevelopmental Disorders Research Center.

\section{References}

American Psychiatric Association. Diagnostic and statistical manual of mental disorders: DSM-IV. 4. Washington, DC: APA; 1994.

Baxter LR Jr, Phelps ME, Mazziotta JC, Guze BH, Schwartz JM, Selin CE. Local cerebral glucose metabolic rates in obsessive-compulsive disorder: A comparison with rates in unipolar depression and in normal controls. Archives of General Psychiatry. 1987; 44(3):211-218. [PubMed: 3493749]

Beckmann CF, Jenkinson M, Smith SM. General multilevel linear modeling for group analysis in FMRI. Neuroimage. 2003; 20(2):1052-1063. [PubMed: 14568475]

Bodfish, JW.; Symons, FW.; Lewis, MH. The Repetitive Behavior Scale-Revised. Morganton, NC: Western Carolina Center Research Reports; 1999.

Braunholtz DA, Edwards SJ, Lilford RJ. Are randomized clinical trials good for us (in the short term)? Evidence for a 'trial effect'. Journal of Clinical Epidemiology. 2001; 54(3):217-224. [PubMed: 11223318]

Buchsbaum MS, Hollander E, Haznedar MM, Tang C, Spiegel-Cohen J, Wei TC, Solimando A, Buchsbaum BR, Robins D, Bienstock C, Cartwright C, Mosovich S. Effect of fluoxetine on regional cerebral metabolism in autistic spectrum disorders: A pilot study. International Journal of Neuropsychopharmacology. 2001; 4(2):119-125. [PubMed: 11466160]

Cummings JL. Frontal-subcortical circuits and human behavior. Archives of Neurology. 1993; 50(8): 873-880. [PubMed: 8352676]

Devinsky O, Morrell MJ, Vogt BA. Contributions of anterior cingulate cortex to behaviour. Brain. 1995; 118(Pt 1):279-306. [PubMed: 7895011] 
Dichter GS, Felder JN, Bodfish JW. Autism is characterized by dorsal anterior cingulate hyperactivation during social target detection. Society of Cognitive and Affective Neuroscience. 2009; 4(3):215-226.

Drewe EA. Go-no go learning after frontal lobe lesions in humans. Cortex. 1975; 11(1):8-16. [PubMed: 1149469]

Forbes EE, Hariri AR, Martin SL, Silk JS, Moyles DL, Fisher PM, Brown SM, Ryan ND, Birmaher B, Axelson DA, Dahl RE. Altered striatal activation predicting real-world positive affect in adolescent major depressive disorder. American Journal of Psychiatry. 2009; 166(1):64-73. [PubMed: 19047324]

Gomot M, Belmonte MK, Bullmore ET, Bernard FA, Baron-Cohen S. Brain hyper-reactivity to auditory novel targets in children with high-functioning autism. Brain. 2008; 131(Pt 9):2479_ 2488. [PubMed: 18669482]

Gu BM, Park JY, Kang DH, Lee SJ, Yoo SY, Jo HJ, Choi CH, Lee JM, Kwon JS. Neural correlates of cognitive inflexibility during task-switching in obsessive-compulsive disorder. Brain. 2008; 131(Pt 1):155-164. [PubMed: 18065438]

Guy, W. DHEW Pub No (ADM). 1976. ECDEU Assessment Manual for PsychopharmacologyRevised; p. 76-338.

Hollander E, Phillips A, Chaplin W, Zagursky K, Novotny S, Wasserman S, Iyengar R. A placebo controlled crossover trial of liquid fluoxetine on repetitive behaviors in childhood and adolescent autism. Neuropsychopharmacology. 2005; 30(3):582-589. [PubMed: 15602505]

Insel TR. Toward a neuroanatomy of obsessive-compulsive disorder. Archives of General Psychiatry. 1992; 49(9):739-744. [PubMed: 1514879]

Jenkinson M, Bannister P, Brady M, Smith S. Improved optimization for the robust and accurate linear registration and motion correction of brain images. Neuroimage. 2002; 17(2):825-841. [PubMed: 12377157]

Jenkinson M, Smith S. A global optimisation method for robust affine registration of brain images. Medical Image Analysis. 2001; 5(2):143-156. [PubMed: 11516708]

Just MA, Cherkassky VL, Keller TA, Kana RK, Minshew NJ. Functional and anatomical cortical underconnectivity in autism: Evidence from an FMRI study of an executive function task and corpus callosum morphometry. Cerebral Cortex. 2007; 17(4):951-961. [PubMed: 16772313]

Kanner L. Autistic disturbances of affective contact. Nervous Child. 1943; 2:217-250.

King BH, Hollander E, Sikich L, McCracken JT, Scahill L, Bregman JD, Donnelly CL, Anagnostou E, Dukes K, Sullivan L, Hirtz D, Wagner A, Ritz L. Lack of efficacy of citalopram in children with autism spectrum disorders and high levels of repetitive behavior: Citalopram ineffective in children with autism. Archives of General Psychiatry. 2009; 66(6):583-590. [PubMed: 19487623]

Lam KS, Aman MG. The Repetitive Behavior Scale-Revised: Independent validation in individuals with autism spectrum disorders. Journal of Autism and Developmental Disorders. 2007; 37(5): 855-866. [PubMed: 17048092]

Langen M, Schnack HG, Nederveen H, Bos D, Lahuis BE, de Jonge MV, van Engeland H, Durston S. Changes in the developmental trajectories of striatum in autism. Biological Psychiatry. 2009; 66(4):327-333. [PubMed: 19423078]

Lewis MH, Bodfish JW. Repetitive behavior disorders in autism. Mental Retardation \& Developmental Disabilities Research Reviews. 1998; 4:80-89.

Lewis MH, Gluck JP, Beauchamp AJ, Keresztury MF, Mailman RB. Long-term effects of early social isolation in Macaca mulatta: Changes in dopamine receptor function following apomorphine challenge. Brain Research. 1990; 513(1):67-73. [PubMed: 1693540]

Lord C, Risi S, Lambrecht L, Cook EH Jr, Leventhal BL, DiLavore PC, Pickles A, Rutter M. The autism diagnostic observation schedule-generic: A standard measure of social and communication deficits associated with the spectrum of autism. Journal of Autism \& Developmental Disorders. 2000; 30(3):205-223. [PubMed: 11055457]

MacDonald AW 3rd, Cohen JD, Stenger VA, Carter CS. Dissociating the role of the dorsolateral prefrontal and anterior cingulate cortex in cognitive control. Science. 2000; 288(5472):1835-1838. [PubMed: 10846167] 
Manoach DS. Prefrontal cortex dysfunction during working memory performance in schizophrenia: Reconciling discrepant findings. Schizophrenia Research. 2003; 60(2-3):285-298. [PubMed: 12591590]

Marsh R, Zhu H, Wang Z, Skudlarski P, Peterson BS. A developmental fMRI study of self-regulatory control in Tourette's syndrome. American Journal of Psychiatry. 2007; 164(6):955-966. [PubMed: 17541057]

Masterman DL, Cummings JL. Frontal-subcortical circuits: The anatomic basis of executive, social and motivated behaviors. Journal of Psychopharmacology. 1997; 11(2):107-114. [PubMed: 9208374]

McDougle CJ, Naylor ST, Cohen DJ, Volkmar FR, Heninger GR, Price LH. A double-blind, placebocontrolled study of fluvoxamine in adults with autistic disorder. Archives of General Psychiatry. 1996; 53(11):1001-1008. [PubMed: 8911223]

Robbins, TW. Integrating the neurobiological and neuropsychlogical dimensions of autism. In: Russell, J., editor. Autism as an executive disorder. Oxford: Oxford University Press; 1997. p. 22-53.

Robbins, TW.; Mittleman, G.; O’Brien, J.; Winn, P. Neuropsychological significance of stereotypy induced by stimulant drugs. In: Cooper, SJ.; Dourish, C., editors. Neurobiology of behavioral stereotypy. Oxford: Oxford University Press; 1990. p. 22-63.

Russell, J. Autism as an executive disorder. Oxford: Oxford University Press; 1997.

Scahill L, McDougle CJ, Williams SK, Dimitropoulos A, Aman MG, McCracken JT, et al. Children's Yale-Brown Obsessive Compulsive Scale Modified for Pervasive Developmental Disorders. Journal of the American Academy of Child and Adolescent Psychiatry. 2006; 45(9):1114-1123. [PubMed: 16926619]

Schmitz N, Rubia K, Daly E, Smith A, Williams S, Murphy DG. Neural correlates of executive function in autistic spectrum disorders. Biological Psychiatry. 2006; 59(1):7-16. [PubMed: 16140278]

Shafritz KM, Dichter GS, Baranek GT, Belger A. The neural circuitry mediating shifts in behavioral response and cognitive set in autism. Biological Psychiatry. 2008; 63(10):974-980. [PubMed: 17916328]

Singer HS, Hahn IH, Moran TH. Abnormal dopamine uptake sites in postmortem striatum from patients with Tourette's syndrome. Annals of Neurology. 1991; 30(4):558-562. [PubMed: 1838678]

Smith SM. Fast robust automated brain extraction. Human Brain Mapping. 2002; 17(3):143-155. [PubMed: 12391568]

Smith SM, Jenkinson M, Woolrich MW, Beckmann CF, Behrens TEJ, Johansen-Berg H, et al. Advances in functional and structural MR image analysis and implementation as FSL. NeuroImage. 2004; 23(Suppl 1):S208-219. [PubMed: 15501092]

Thakkar KN, Polli FE, Joseph RM, Tuch DS, Hadjikhani N, Barton JJ, Manoach DS. Response monitoring, repetitive behaviour and anterior cingulate abnormalities in ASD. Brain. 2008; 131(Pt 9):2464-2478. [PubMed: 18550622]

Visser JE, Bar PR, Jinnah HA. Lesch-Nyhan disease and the basal ganglia. Brain Research Reviews. 2000; 32(2-3):449-475. [PubMed: 10760551]

Weschler, D. Weschler Abbreviated Scale of Intelligence (WASI). San Antonio, TX: Harcourt Assessment; 1999.

Woolrich MW, Ripley BD, Brady M, Smith SM. Temporal autocorrelation in univariate linear modeling of FMRI data. Neuroimage. 2001; 14(6):1370-1386. [PubMed: 11707093] 

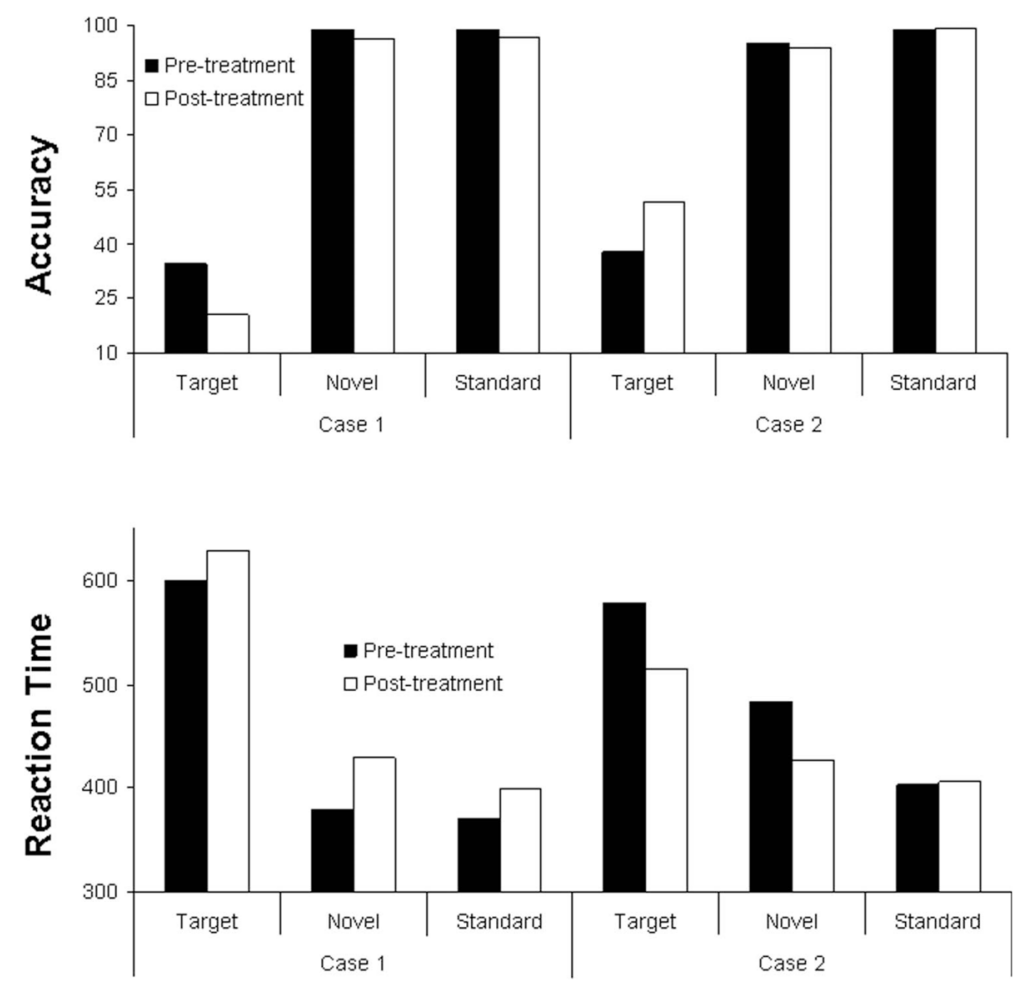

Figure 1.

fMRI Task Performance for both participants pre- and post-treatment. 


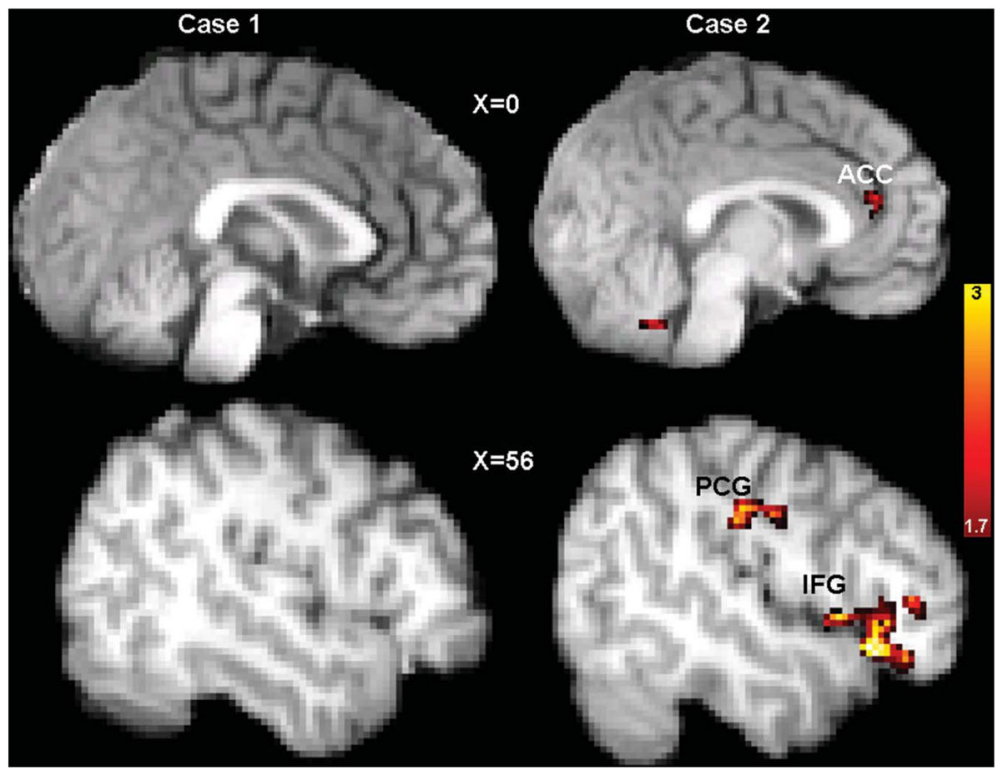

Figure 2.

Activation maps depicting areas of statistically increased activation after citalopram in response to target events in Case 1, a citalopram-treatment non-responder (left), and Case 2, a citalopram-treatment responder (right). Activations are displayed superimposed on each participant's own structural brain image. 


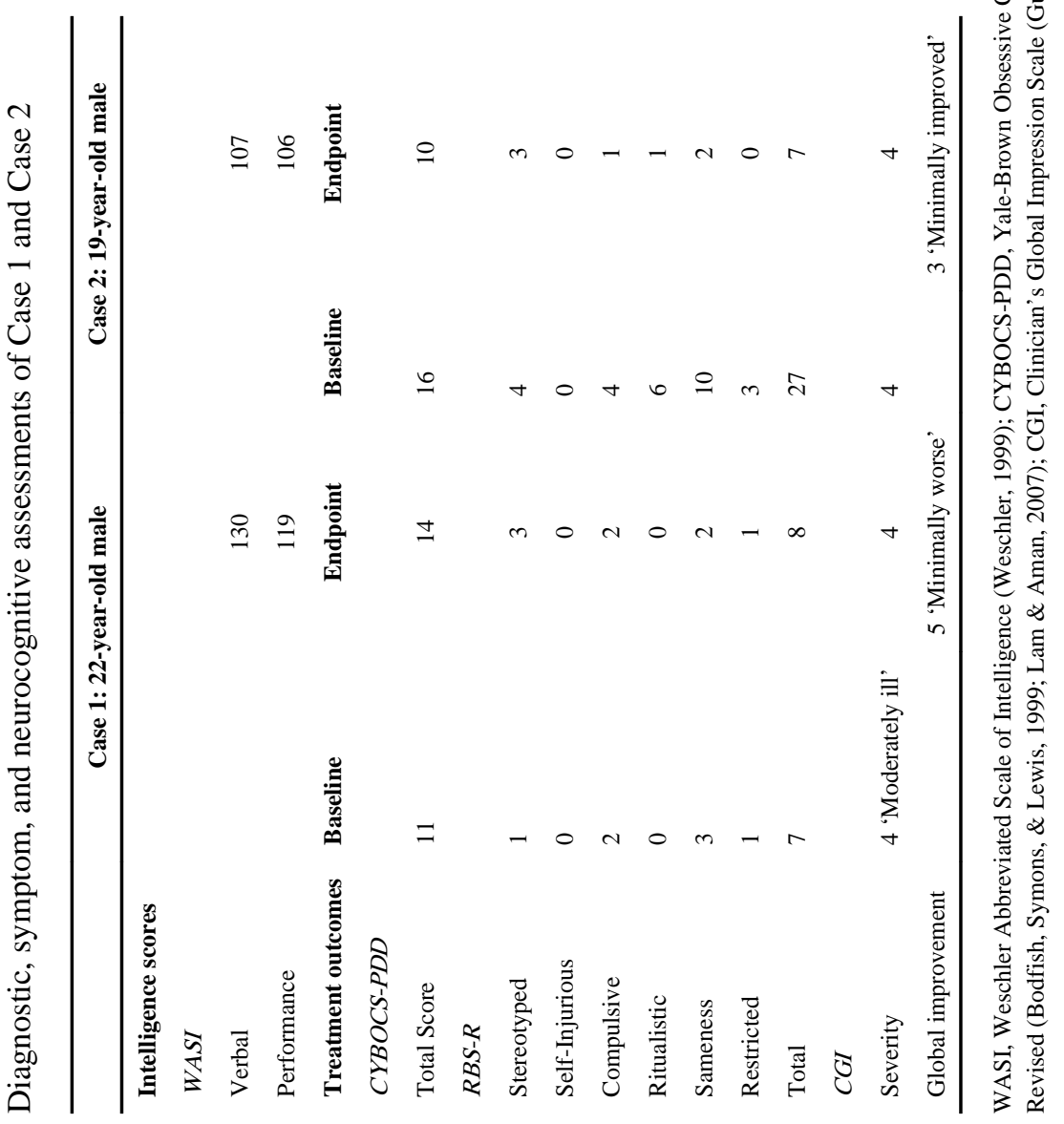




\section{TABLE 2}

Regions of increased activation, in Montreal Neurologic Institute (MNI) coordinates, in response to oddball target stimuli in Case 1, who showed increases in repetitive behaviors after citalopram treatment, and Case 2 , who showed reductions in repetitive behaviors after citalopram treatment

\begin{tabular}{lrrrr} 
& \multicolumn{3}{l}{ MNI Coordinates (mm) } & \\
\cline { 2 - 4 } Region & $\mathbf{X}$ & $\mathbf{Y}$ & $\mathbf{Z}$ & $\mathbf{Z}$ max \\
\hline Case 1 & & & & \\
Supplementary motor cortex & 10 & -4 & 48 & 2.20 \\
Right occipital pole & 16 & -98 & 8 & 1.79 \\
Right postcentral gyrus & -60 & -20 & 30 & 1.74 \\
Case 2 & & & & \\
Right inferior frontal gyrus & 56 & 24 & -10 & 2.61 \\
Anterior cingulate cortex (ACC) & 2 & 38 & 16 & 2.01 \\
Left supramarginal gyrus & -54 & -32 & 30 & 1.99 \\
Left frontal pole & -20 & 50 & 2 & 1.86 \\
Left inferior frontal gyrus & -36 & 24 & -4 & 1.82 \\
Left fusiform gyrus & -10 & -92 & -24 & 1.79 \\
Left middle frontal gyrus & -40 & 38 & 12 & 1.76 \\
Superior frontal gyrus & 4 & 54 & 28 & 1.64 \\
Right post-central gyrus & 54 & -18 & 32 & 1.63 \\
\hline
\end{tabular}

